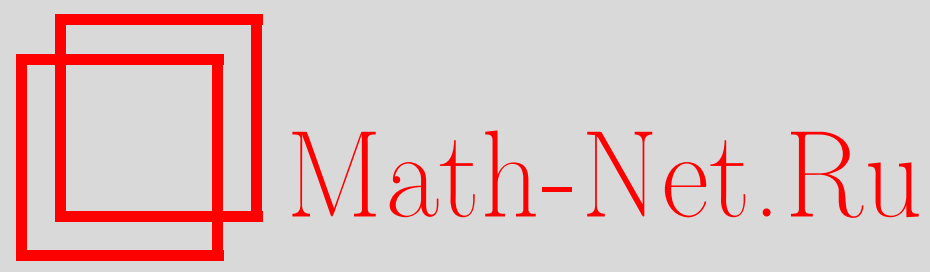

В. В. Кучеренко, Н. Л. Василевский, Оператор сдвига, порожденный тригонометрической системой, Матем. заметкu, 2000, том 67, выпуск 4, 539-548

DOI: https://doi.org/10.4213/mzm869

Использование Общероссийского математического портала Math-Net.Ru подразумевает, что вы прочитали и согласны с пользовательским соглашением http://www . mathnet.ru/rus/agreement

Параметры загрузки:

IP : 3.85 .183 .62

26 апреля 2023 г., $17: 52: 38$

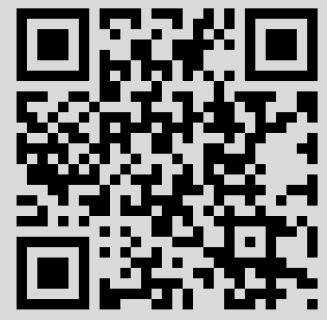




\title{
ОПЕРАТОР СДВИГА, ПОРОЖДЕННЫЙ ТРИГОНОМЕТРИЧЕСКОЙ СИСТЕМОЙ
}

\author{
В. В. Кучеренко, Н. Л. Василевский
}

В работе изучается изометрический оператор, порожденный тригонометрической системой. Этот оператор используется в задачах с косой производной и в теории рядов Фурье. Доказано, что этот изометрический оператор является оператором чистого сдвига, и дано применение этого факта к обратной задаче магнитопроводности.

Библиографияя: 9 названий.

\section{§1. Постановка задачи}

Рассмотрим в пространстве $L_{2}[0,1]$ две полные ортонормированные системы функций

$$
\{1 ; \sqrt{2} \cos (\pi n \eta), n=1,2, \ldots\}, \quad\{\sqrt{2} \sin (\pi n \eta), n=1,2, \ldots\} .
$$

Определим на $L_{2}[0,1]$ линейньй оператор $D$ соотношением

$$
D f \stackrel{\text { def }}{=} 2 \sum_{n=1}^{\infty} \cos (\pi n \eta) \int_{0}^{1} \sin (\pi n \xi) f(\xi) d \xi
$$

УТВЕРЖДЕНИЕ 1. 1. Оператор D изометричен.

2. Ортогональное дополнение $\kappa$ области значений $R(D)$ одномерно и состоит из констант.

3. Справедливы соотношения

$$
D^{*} f=2 \sum_{n=1}^{\infty} \sin (\pi n \eta) \int_{0}^{1} \cos (\pi n \xi) f(\xi) d \xi, \quad D^{*} D=I, \quad D D^{*}=I-P
$$

где $P$ есть ортогональный проектор на функиию $f(x) \equiv 1$, m.e. $P g \stackrel{\text { def }}{=} \int_{0}^{1} g(x) d x$.

Работа выполнена при финансовой поддержке фонда CONACYT (Мексика), грант № 27934Е. 
ДокАЗАТЕЛЬСТВо. 1. Очевидно, для произвольной функции $f(\eta) \in L_{2}[0,1]$ имеем

$$
f(\eta)=2 \sum_{n=1}^{\infty} \sin (\pi n \eta) \int_{0}^{1} \sin (\pi n \xi) f(\xi) d \xi
$$

Поэтому в силу ортонормированности систем (1) получим

$$
(D f, D g)=(f, g)
$$

т.е. $D$ - изометрический оператор.

2. Далее, для произвольного элемента $x=\left\{x_{1}, x_{2}, \ldots\right\} \in \ell_{2}$ функция

$$
f(\eta) \stackrel{\text { def }}{=} \sqrt{2} \sum_{j=1}^{\infty} x_{j} \sin (\pi j \eta) \in L_{2}[0,1] .
$$

Очевидно,

$$
D f=\sqrt{2} \sum_{n=1}^{\infty} x_{n} \cos (\pi n \xi), \quad x \in \ell_{2},
$$

и ортогональное дополнение к функциям вида (5) в силу полноты системы

$$
\{1 ; \sqrt{2} \cos (\pi n \xi), n=1,2, \ldots\}
$$

есть одномерное подпространство констант.

3. Формула (3) для оператора $D^{*}$ с очевидностью следует из формулы (2). Очевидно, изометрический оператор $D$ удовлетворяет соотношениям: $D^{*} D=I, D D^{*}=I-P$, где $P$ - ортопроектор на подпространство $R(D)^{\perp}$. Из пункта 2 следует, что $R^{\perp}(D)$ есть одномерное подпространство констант (постоянных функций на $[0,1]$ ).

Рассмотрим функции $\varphi_{k}(\eta) \stackrel{\text { def }}{=} D^{k} 1, k=0,1, \ldots$ Пусть $L_{D}$ есть замыкание линейной оболочки векторов $\varphi_{k}(\eta)$ в $L_{2}[0,1]$, а $L_{D}^{\perp}$ - его ортогональное дополнение: $L_{2}[0,1]=$ $L_{D} \oplus L_{D}^{\perp}$.

ЛЕмма 1. 1. Подпространства $L_{D}, L_{D}^{\perp}$ инвариантны относительно операторов $D, D^{*}$. Функиии $\left\{\varphi_{k}(\eta), k=0,1, \ldots\right\}$ образуют ортонормированную систему.

2. Ограничение $\left.D_{s} \stackrel{\text { def }}{=} D\right|_{L_{D}}$ есть оператор чистого сдвига.

3. Ограничение $\left.D_{U} \stackrel{\text { def }}{=} D\right|_{L_{D}}$ ecть унитарный оператор.

ДоказАТЕЛьство. 1. Имеем $\left(\varphi_{k}, \varphi_{j}\right)=\left(D^{k} 1, D^{j} 1\right)$. Пусть, например, $k \geqslant j$. Тогда в силу равенства $D^{*} D=I$ получим

$$
\left(\varphi_{k}, \varphi_{j}\right)=\left(D^{k-j} 1,1\right)=\left(1,\left(D^{*}\right)^{k-j} 1\right)
$$

Отсюда в силу равенства $D^{*} 1=0$ и соотношения $(1,1)=1$ получаем, что $\left(\varphi_{k}, \varphi_{j}\right)=\delta_{k j}$.

2. Элементы подпространства $L_{D}$ представимы в виде ряда Фурье:

$$
f=\sum_{k=1}^{\infty} c_{k} \varphi_{k}, \quad \sum_{k=1}^{\infty}\left|c_{k}\right|^{2}<\infty
$$


Поскольку $D \varphi_{k}=\varphi_{k+1}$, то, очевидно, $D L_{D} \subseteq L_{D}$.

Далее, ввиду равенств $D^{*} D=I, D^{*} 1=0$ имеем

$$
D^{*} f=\sum_{k=1}^{\infty} c_{k} \varphi_{k-1}, \quad \text { т.e. } D^{*} L_{D} \subseteq L_{D}
$$

Очевидно, что на подпространстве $L_{D}$ оператор $D$ унитарно эквивалентен оператору чистого сдвига: $U^{*} D U\left\{c_{1}, c_{2}, \ldots\right\}=\left\{0, c_{1}, c_{2}, \ldots\right\}$.

3. Теперь докажем инвариантность $L \frac{\perp}{D}$ относительно $D, D^{*}$. Пусть $f \in L_{D}^{\perp}$, т.е. $\left(f, D^{k} 1\right)=0, k=0,1, \ldots$ Тогда $\left(D f, D^{k} 1\right)=\left(f, D^{k-1} 1\right)=0$ при $k \geqslant 1$ и $(D f, 1)=$ $\left(f, D^{*} 1\right)=0$, ибо $D^{*} 1=0$. Следовательно, $D f$ также принадлежит $L_{D}$.

Аналогично получим, что $D^{*} L_{D}^{\perp} \subset L_{D}^{\perp}$.

Покажем, что $\left.D\right|_{L} \frac{\perp}{D}$ унитарен. Поскольку $D$ изометричен, то для унитарности его ограничения на $L \frac{\perp}{D}$ достаточно доказать, что $D L \frac{\perp}{D}=L \frac{\perp}{D}$. Допустим противное. Тогда в $L \frac{\perp}{D}$ существует элемент $g \neq 0$ такой, что $\left(D L_{D}^{\perp}, g\right)=0$. Значит, $\left(L_{D}^{\perp}, D^{*} g\right)=0$. Поэтому в силу разложения $L_{2}=L_{D} \oplus L_{D}^{\frac{1}{D}}$ имеем включение $D^{*} g \subset L_{D}$. Из инвариантности $L_{D}$ относительно оператора $D$ и равенства $D D^{*}=I-P$ следует, что $(g-P g) \in L_{D}$. Поскольку $P g=(g, 1) \in L_{D}$, то, следовательно, $g \in L_{D}$. Это противоречит включению $g \in L_{D}^{\perp}$. Полученное противоречие доказьвает, что $D L_{D}^{\perp}=L_{D}^{\perp}$, а значит, $\left.D\right|_{L_{D}}$ есть унитарньй оператор.

Пункт 3 леммы есть уточнение разложения Уолда [1] в случае одномерного подпространства $R(D)^{\perp}$.

Оператор $D$, очевидно, можно определить для двух произвольных полных ортонормированных систем, однако для тригонометрических систем (1) он обладает следующим нетривиальным свойством.

Теорема. Функиии $D^{k} 1, k=0,1, \ldots$, образуют ортонормированный базис в $L_{2}[0,1]$, а значит, оператор D унитарно әквивалентен оператору чистого сдвига.

Доказательство теоремы приводится в следующем параграфе. Оно основано на интегральном представлении функций $\varphi_{k}(\eta)$ через степени сингулярного интегрального оператора с ядром типа Гильберта [2].

ЛЕмма 2. На функииях из $L_{2}[0,1]$ справедливо представление

$$
(D g)(\eta)=\int_{0}^{1} g(\xi) \frac{\sin (\pi \xi)}{\cos (\pi \eta)-\cos (\pi \xi)} d \xi,
$$

где интеграл понимается в смысле главного значения.

ДоказАтельСтво. Рассмотрим сумму

$$
2 \sum_{n=1}^{N} \sin (\pi n \xi) \cos (\pi n \eta)=\sum_{n=1}^{N} \sin [\pi n(\eta+\xi)]-\sum_{n=1}^{N} \sin [\pi n(\eta-\xi)] .
$$

Как известно,

$$
\sum_{n=1}^{N} \sin (n \alpha)=\frac{\sin ^{2}((N+1) \alpha / 2)}{\sin (\alpha / 2)} \cos \frac{\alpha}{2}-\frac{1}{2} \sin [(N+1) \alpha] .
$$


Следовательно,

$$
\begin{aligned}
& 2 \sum_{n=1}^{N} \sin (\pi n \xi) \cos (\pi n \eta)=\frac{\sin ^{2}[(N+1)(\xi+\eta) \pi / 2]}{\sin [\pi(\xi+\eta) / 2]} \cos \frac{\pi(\xi+\eta)}{2}-\frac{1}{2} \sin [\pi(N+1)(\xi+\eta)] \\
& \quad-\frac{\sin ^{2}[(N+1)(\eta-\xi) \pi / 2]}{\sin [\pi(\eta-\xi) / 2]} \cos \frac{\pi(\eta-\xi)}{2}+\frac{1}{2} \sin [\pi(N+1)(\eta-\xi)] .
\end{aligned}
$$

Заметим, что ограниченные интегральные операторы с ядрами $\sin [\pi(N+1)(\eta \pm \xi)]$ при $N \rightarrow+\infty$ слабо сходятся к нулю на пространстве $L_{2}[0,1]$.

Для функций $f(\xi) \in L_{2}[0,1]$, равных нулю в окрестности точек $\xi=0,1$, нетрудно убедиться в справедливости соотношения

$$
\lim _{N \rightarrow \infty} \int_{0}^{1} f(\xi) \frac{\sin ^{2}[(N+1) \pi(\xi+\eta) / 2]}{\sin [\pi(\xi+\eta) / 2]} \cos \frac{\pi(\xi+\eta)}{2} d \xi=\frac{1}{2} \int_{0}^{1} f(\xi) \frac{\cos [\pi(\xi+\eta) / 2]}{\sin [\pi(\xi+\eta) / 2]} d \xi
$$

Далее, для непрерывно дифференцируемых функций, равных нулю в окрестности точек $\xi=0,1$, прямыми вычислениями убеждаемся в справедливости предельных равенств

$$
\lim _{N \rightarrow \infty} \int_{0}^{1} f(\xi) \frac{\sin ^{2}[(N+1) \pi(\eta-\xi) / 2]}{\sin [\pi(\eta-\xi) / 2]} \cos \frac{\pi(\eta-\xi)}{2} d \xi=\frac{1}{2} \int_{0}^{1} f(\xi) \frac{\cos [\pi(\eta-\xi) / 2]}{\sin [\pi(\eta-\xi) / 2]} d \xi
$$

Резюмируя, получим, что на множестве $K[0,1]$, состоящем из непрерьвно дифференцируемых функций, обращающихся в нуль в окрестности точек $\xi=0,1$, справедливо представление

$$
D f=\frac{1}{2} \int_{0}^{1} f(\xi)\left\{\operatorname{ctg} \frac{\pi(\eta+\xi)}{2}-\operatorname{ctg} \frac{\pi(\eta-\xi)}{2}\right\} d \xi .
$$

Из соотношения (14) приведением подобных членов получим, что на $K[0,1]$ справедливо соотношение (8).

Поскольку оператор $D$ изометричен, то в силу [3], [4] предельным переходом соотношение (8) распространяется со множества $K[0,1]$ на все $L_{2}[0,1]$.

Интегральное представление (8) заменой переменных приводится к интегралу с ядром Гильберта $(x-t)^{-1}$. С этой целью положим $\eta=\pi^{-1} \arccos x, \xi=\pi^{-1} \arccos t$. Очевидно, что

$$
(D g)\left(\pi^{-1} \arccos x\right)=\frac{1}{\pi} \int_{-1}^{1} g\left(\pi^{-1} \arccos t\right)(x-t)^{-1} d t .
$$

Теперь функция $\widetilde{g}(x) \stackrel{\text { def }}{=} g\left(\pi^{-1} \arccos x\right)$, где $g(t) \in L_{2}[0,1]$, принадлежит пространству $L_{2}([-1,1], \rho)$ с весом $\rho(x) \stackrel{\text { def }}{=}\left(1-x^{2}\right)^{-1 / 2}$.

Поэтому из изометричности оператора $D$ в пространстве $L_{2}[0,1]$ следует, что оператор

$$
(A f)(x) \stackrel{\text { def }}{=} \frac{1}{\pi} \int_{-1}^{1} f(t)(x-t)^{-1} d t
$$

изометричен в пространстве $L_{2}([-1,1], \rho)$. 
Положим $\widetilde{\varphi}_{k}(x) \stackrel{\text { def }}{=} \varphi_{k}\left(\pi^{-1} \arccos x\right)$, где $\varphi_{k}(\eta) \stackrel{\text { def }}{=} D^{k} 1$. Очевидно, что $\widetilde{\varphi}_{k}(x) \in$ $L_{2}([-1,1], \rho)$ и

$$
\widetilde{\varphi}_{k}(x)=A \widetilde{\varphi}_{k-1}, \quad k \geqslant 1, \quad \varphi_{0}(x) \stackrel{\text { def }}{=} 1 .
$$

Следуя [2], сведем оператор (16) к оператору на полуоси заменой $x=(z-1) /(z+1)$, $t=(s-1) /(s+1), z, s \in \mathbb{R}_{+}^{1}$. В результате для функций $\bar{\varphi}_{k}(z) \stackrel{\text { def }}{=} \widetilde{\varphi}_{k}((z-1) /(z+1))$ получим выражение

$$
\frac{\bar{\varphi}_{k}(z)}{1+z}=\frac{1}{\pi} \int_{0}^{+\infty} \frac{\bar{\varphi}_{k-1}(s)}{1+s} \frac{d s}{z-s}, \quad \bar{\varphi}_{0} \stackrel{\text { def }}{=} 1 .
$$

Очевидно, функции $\bar{\varphi}_{k}(z)$ принадлежат пространству $L_{2}\left(\mathbb{R}_{+}, \bar{\rho}\right)$ с весом $\bar{\rho}(z) \stackrel{\text { def }}{=}$ $z^{-1 / 2}(1+z)^{-1}$ и ортогональны с этим весом.

\section{§ 2. Доказательство теоремы}

Очевидно, утверждение теоремы эквивалентно плотности линейной оболочки функций $\bar{\varphi}_{k}(z)\left(\right.$ см. (18)) в пространстве $L_{2}\left(\mathbb{R}_{+}^{1}, \bar{\rho}\right)$. Для доказательства этого факта воспользуемся преобразованием Меллина.

1. Представление функций $\bar{\varphi}_{k}(z) /(1+x)$ через преобразование Меллина. Воспользуемся формулами для прямого и обратного преобразования Меллина, определяющими изометрическое отображение $L_{2}\left(\mathbb{R}^{+}\right)$на $L_{2}(\mathbb{R})$ :

$$
\widehat{f}(\lambda) \stackrel{\text { def }}{=}(2 \pi)^{-1 / 2} \int_{0}^{+\infty} x^{-i \lambda-1 / 2} f(x) d x, \quad f(x) \stackrel{\text { def }}{=}(2 \pi)^{-1 / 2} \int_{-\infty}^{+\infty} x^{i \lambda-1 / 2} \widehat{f}(\lambda) d \lambda .
$$

Используя выражение (18), в силу формул работы [2] получим интегральное представление

$$
\frac{\bar{\varphi}_{k}(x)}{1+x}=\frac{(-1)^{k}}{\sqrt{2 \pi}} \int_{-\infty}^{+\infty} x^{i \lambda-1 / 2}\{\operatorname{th}(\pi \lambda)\}^{k} g_{0}(\lambda) d \lambda
$$

где

$$
g_{0}(\lambda) \stackrel{\text { def }}{=} \frac{1}{\sqrt{2 \pi}} \int_{0}^{+\infty} t^{-i \lambda-1 / 2}(1+t)^{-1} d t .
$$

УТВЕРЖДЕНИЕ 2. 1. Имеет место представление

$$
g_{0}(\lambda)=\frac{\sqrt{2 \pi} i e^{\pi(\lambda-i / 2)}}{1-e^{2 \pi(\lambda-i / 2)}}
$$

2. Функиия $g_{0}(\lambda-i / 4) / g_{0}(\lambda+i / 4)$ на прямой $\{\lambda: \operatorname{Im} \lambda=0\}$ по модулю равна 1 и определяется формулой

$$
\frac{g_{0}(\lambda-i / 4)}{g_{0}(\lambda+i / 4)}=-i \frac{1+i e^{2 \pi \lambda}}{1-i e^{2 \pi \lambda}}
$$


ДоказАтЕльство. Рассмотрим, например, случай $\operatorname{Re} \lambda \leqslant 0,|\operatorname{Im} \lambda| \leqslant 1 / 2-\delta$. Из формулы (21) следует, что на дуге окружности $t=R e^{i \varphi}, 0 \leqslant \varphi \leqslant \pi$, подьнтегральная функция имеет порядок $R^{-(1+\delta)}$ и, значит, интеграл по дуге окружности от функции $t^{-i \lambda-1 / 2} /(1+t)$ стремится к нулю при $R \rightarrow+\infty$. Поэтому, поворачивая контур интегрирования $[0,+\infty)$ на угол $2 \pi$, получим равенство

$$
\frac{1}{\sqrt{2 \pi}} \int_{0}^{+\infty} \frac{t^{-i \lambda-1 / 2}}{(1+t)} d t=\sqrt{2 \pi} i e^{\pi(\lambda-i / 2)}+\frac{1}{\sqrt{2 \pi}} e^{2 \pi(\lambda-i / 2)} \int_{0}^{+\infty} \frac{t^{-i \lambda-1 / 2}}{1+t} d t
$$

В результате $k$ вращений контура интегрирования, т.е. при повороте на угол $2 \pi k$, аналогично получим соотношение

$$
\begin{aligned}
\frac{1}{\sqrt{2 \pi}} \int_{0}^{+\infty} \frac{t^{-i \lambda-1 / 2}}{1+t} d t= & \sqrt{2 \pi} i \sum_{n=0}^{k-1} e^{\pi(\lambda-i / 2)+2 \pi n(\lambda-i / 2)} \\
& +\frac{1}{\sqrt{2 \pi}} e^{2 \pi k(\lambda-i / 2)} \int_{0}^{+\infty} \frac{t^{-i \lambda-1 / 2}}{1+t} d t
\end{aligned}
$$

Переходя к пределу в $(25)$ при $k \rightarrow+\infty$ в области $\operatorname{Re} \lambda \leqslant 0,|\operatorname{Im} \lambda| \leqslant 1 / 2-\delta$, получим равенство

$$
\frac{1}{\sqrt{2 \pi}} \int_{0}^{+\infty} \frac{t^{-i \lambda-1 / 2}}{1+t} d t=\sqrt{2 \pi} i \sum_{n=0}^{+\infty} e^{\pi(\lambda-i / 2)+2 \pi n(\lambda-i / 2)}
$$

В силу формулы для суммы членов геометрической прогрессии отсюда следует формула $(22)$ в области $\operatorname{Re} \lambda \leqslant 0,|\operatorname{Im} \lambda| \leqslant 1 / 2-\delta$.

В области $\{\lambda: \operatorname{Re} \lambda>0,|\operatorname{Im} \lambda|<1 / 2-\delta\}$ вращением контура интегрирования против часовой стрелки мы также получаем формулу (22). Из формулы (21) непосредственно следует, что функция $g_{0}(\lambda)$ аналитична в области $\{\lambda:|\operatorname{Im} \lambda|<1 / 2\}$. Поэтому, используя аналитическое продолжение, получим формулу (22) во всей комплексной плоскости. Формула (23) прямо следует из формулы (22).

Из утверждения 2 непосредственно следует

УТВЕРЖДЕНИЕ 3. 1. Функиии $\{\operatorname{th}(\pi \lambda)\}^{k} g_{0}(\lambda), k=0,1, \ldots$, аналитичны в полосе $|\operatorname{Im} \lambda|<1 / 2-\varepsilon, \varepsilon>0$, и удовлетворяют в этой полосе оченке

$$
\left|\{\operatorname{th}(\pi \lambda)\}^{k} g_{0}(\lambda)\right| \leqslant C(\varepsilon, k) e^{-\pi|\lambda|}
$$

2. В интегральном представлении (20) в качестве контура интегрирования мохсно взять произвольную прямую $\lambda=s+i \sigma, s \in \mathbb{R}^{1},|\sigma|<1 / 2$, параллельную вещественной оси.

2. Доказательство плотности линейной оболочки функций $\bar{\varphi}_{k}(z)$ в пространстве $L_{2}\left(\mathbb{R}_{+}^{1}, \bar{\rho}\right)$ с весом $\bar{\rho}=z^{-1 / 2}(1+z)^{-1}$. Плотность системы $\bar{\varphi}_{k}(z)$ эквивалентна утверждению, что из условий

$$
\int_{0}^{+\infty} \bar{\varphi}_{k}(z) f^{*}(z) z^{-1 / 2}(1+z)^{-1} d z=0, \quad k=0,1, \ldots
$$


следует, что $f(z)=0$ в пространстве $L_{2}\left(\mathbb{R}_{+}^{1}, \bar{\rho}\right)$. Для $f \in L_{2}\left(\mathbb{R}_{+}^{1}, \bar{\rho}\right)$ положим $f_{\mu} \stackrel{\text { def }}{=}$ $z^{-1 / 4}(1+\mu \sqrt{z})^{-1} f$. Очевидно, для комплексных значений параметра $\mu$ в $\mathbb{C} \backslash\{-\infty, 0\}$ имеем $f_{\mu} \in L_{2}\left(\mathbb{R}_{+}^{1}\right)$. Ясно, что условие (26) полноты системы $\left\{\bar{\varphi}_{k}(z)\right\}$ можно переформулировать так: из равенств

$$
\int_{0}^{+\infty}\left(z^{-1 / 4}+\mu z^{1 / 4}\right) \frac{\bar{\varphi}_{k}(z)}{1+z} f_{\mu}(z) d z=0, \quad k=0,1, \ldots
$$

для функций $f_{\mu} \in L_{2}\left(\mathbb{R}_{+}^{1}\right)$ следует, что $f_{\mu}(z)=0$ в $L_{2}\left(\mathbb{R}_{+}^{1}\right)$.

Далее, изучим условие полноты в форме (27). Сдвигая контур интегрирования в интегральном представлении (20) на $\pm i / 4$, получим равенство

$$
\begin{aligned}
\left(z^{-1 / 4}+\mu z^{1 / 4}\right) \frac{\bar{\varphi}_{k}(z)}{1+z}= & \frac{(-1)^{k}}{\sqrt{2 \pi}} \int_{-\infty}^{+\infty} z^{i \lambda-1 / 2}\left\{\left[\operatorname{th}\left(\pi\left(\lambda+\frac{i}{4}\right)\right)\right]^{k} g_{0}\left(\lambda+\frac{i}{4}\right)\right. \\
& \left.+\mu\left[\operatorname{th}\left(\pi\left(\lambda-\frac{i}{4}\right)\right)\right]^{k} g_{0}\left(\lambda-\frac{i}{4}\right)\right\} d \lambda .
\end{aligned}
$$

Теперь, воспользовавшись унитарностью преобразования Меллина (19) и формулой (28), мы запишем условие полноты (27) в следующей форме: если функция $\widehat{f}(\lambda) \in$ $L_{2}\left(\mathbb{R}_{+}^{1}\right)$ удовлетворяет условию

$$
\begin{gathered}
\int_{-\infty}^{+\infty}\left\{\left[\operatorname{th}\left(\pi\left(\lambda+\frac{i}{4}\right)\right)\right]^{k} g_{0}\left(\lambda+\frac{i}{4}\right)+\mu\left[\operatorname{th}\left(\pi\left(\lambda-\frac{i}{4}\right)\right)\right]^{k} g_{0}\left(\lambda-\frac{i}{4}\right)\right\} \widehat{f}(\lambda) d \lambda=0 \\
k=0,1, \ldots
\end{gathered}
$$

то $\widehat{f}(\lambda)=0$ в $L_{2}\left(\mathbb{R}_{+}^{1}\right)$. Далее, произведем в интеграле $(29)$ замену $\lambda=\lambda(w)$, где $w(\lambda) \stackrel{\text { def }}{=}\left\{1+e^{-2 \pi(\lambda+i / 4)}\right\}^{-1}$. Как известно [5], отображение $w(\lambda)$ переводит вещественную ось в верхнюю половину дуги окружности: $w(\varphi)=1 / 2+e^{i \varphi} / 2, \varphi \in[0,2 \pi)$. При этом [6] $w(+\infty)=1, w(-\infty)=0,2 w(\lambda)-1=\operatorname{th}[\pi(\lambda+i / 4)]$. Следовательно, в результате замены представим интеграл (29) как интеграл по верхней половине дуги окружности, которую в дальнейшем обозначим через $\Gamma$ :

$$
\begin{gathered}
\int_{0}^{\pi}\left\{(2 w-1)^{k} g_{0}\left(\lambda(w)+\frac{i}{4}\right)+\mu\left(2 w^{*}-1\right)^{k} g_{0}\left(\lambda(w)-\frac{i}{4}\right)\right\} \frac{\left.\widehat{f}(\lambda(w))\right|_{\Gamma}}{|w(\varphi)(1-w(\varphi))|} d \varphi=0 \\
k=0,1, \ldots
\end{gathered}
$$

Здесь $\widehat{f}(\lambda(w(\varphi))) / \sqrt{|w(\varphi)(1-w(\varphi))|} \in L_{2}[0, \pi]$. Преобразуем подынтегральное выражение в (30). Из формул $(22),(23)$ получим выражения

$$
\begin{gathered}
\left.\frac{g_{0}(\lambda-i / 4)}{g_{0}(\lambda+i / 4)}\right|_{\Gamma}=i e^{-i \varphi} \\
\left.g_{0}\left(\lambda+\frac{i}{4}\right)\right|_{\Gamma}=-\sqrt{2 \pi i} w(\varphi) \sqrt{\frac{1-w(\varphi)}{w(\varphi)}} .
\end{gathered}
$$


С учетом формул (31), (32) интеграл (30) можно представить в виде

$$
\int_{0}^{\pi}\left\{e^{i k \varphi}+i \mu e^{-i \varphi(k+1)}\right\} \frac{\widehat{f}(\lambda(w(\varphi))) e^{i \psi(\varphi)}}{\sqrt{|w(\varphi)(1-w(\varphi))|}} d \varphi=0, \quad k=0,1, \ldots
$$

Здесь $\psi(\varphi)$ - непрерьвная вещественная функция на $[0, \pi]$. Поскольку

$$
\left.\left|\frac{d \lambda}{d \varphi}\right|\right|_{\Gamma}=\frac{1}{2 \pi|w(\varphi)(1-w(\varphi))|}
$$

то, как отмечено выше, из условия $\widehat{f}(\lambda) \in L_{2}\left(\mathbb{R}^{1}\right)$ следует, что функция $\widehat{f}(\lambda(w(\varphi))) /$ $\sqrt{|w(\varphi)(1-w(\varphi))|}$ принадлежит $L_{2}[0, \pi]$.

Из проведенных преобразований следует, что если система функций $\left\{\bar{\varphi}_{k}(z)\right\}$ не плотна, то существует не равная нулю в $L_{2}\left(\mathbb{R}_{+}^{1}, \bar{\rho}\right)$ функция $f(z)$, удовлетворяющая условиям $(26)$, а значит, не равная нулю в $L_{2}[0, \pi]$ функция $\widehat{f}_{\mu}(\lambda(w(\varphi))) / \sqrt{|w(\varphi)(1-w(\varphi))|}$ ортогональна системе функций

$$
e^{i k \varphi}+i \mu e^{-i \varphi(k+1)}, \quad k=0,1, \ldots, \quad \mu \in \mathbb{C} \backslash\{-\infty, 0\},
$$

на $[0, \pi]$.

В свою очередь, из обратимости проделанных преобразований следует, что из полноты системы (34) вытекает полнота системы $\left\{\bar{\varphi}_{k}(z)\right\}$. Положим значение параметра $\mu=i$. Получим

$$
\begin{gathered}
e^{i \varphi k}-e^{-i \varphi(k+1)}=e^{-i \varphi / 2}\left[e^{i \varphi(k+1 / 2)}-e^{-i \varphi(k+1 / 2)}\right]=2 i e^{-i \varphi / 2} \sin \left(k+\frac{1}{2}\right) \varphi \\
k=0,1, \ldots
\end{gathered}
$$

Как хорошо известно, система $\sin (k+1 / 2) \varphi, k=0,1, \ldots$, полна в $L_{2}[0, \pi]$. Следовательно, система (35), а значит, и система $\left\{\bar{\varphi}_{k}(z)\right\}$ полны. Теорема доказана.

ЗАмЕчАниЕ. Поскольку мы доказали, что полнота системы $\left\{\bar{\varphi}_{k}(z)\right\}$ и системы (34) эквивалентны, то из полноты системы $\left\{\bar{\varphi}_{k}(z)\right\}$, в частности, следует полнота систем (34) для всех значений параметра $\mu \in \mathbb{C} \backslash\{-\infty, 0\}$. Полнота систем (34) другим методом была доказана в [7].

\section{§3. Обратная задача магнетосопротивления}

Рассмотрим металлический образец в форме параллелепипеда

$$
V=\{x, y, z: 0 \leqslant x \leqslant a, 0 \leqslant y \leqslant c, 0 \leqslant z \leqslant b\}
$$

в однородном магнитном поле $B$ параллельном оси $y$. Пусть на поверхности параллелепипеда на плоскостях перпендикулярных осям $z$ и $y$ нормальные токи $J_{z}, J_{y}$ равны нулю, а на двух торцах перпендикулярных оси $x$ заданы потенциалы $\varphi_{0}(z), \varphi_{1}(z)$. Итак,

$$
\left.J_{z}\right|_{z=0, b}=0,\left.\quad J_{y}\right|_{y=0, c}=0,\left.\quad \varphi\right|_{x=0}=\varphi_{0}(z),\left.\quad \varphi\right|_{x=a}=\varphi_{1}(z) .
$$


Вектор тока $J$ выражается через электрическое поле и тензор проводимости $\left\|\sigma_{i k}\right\|$ : $J=\left\|\sigma_{i k}\right\| E$.

При изотропном законе дисперсии электронов [8] можно показать, что электрическое поле $E_{y}=0$ и $\left(E_{x}, E_{z}\right)=-\operatorname{grad}_{x, z} \varphi(x, z)$, где потеншиал $\varphi$ удовлетворяет следующей краевой задаче с косой производной в прямоугольнике $V_{1} \stackrel{\text { def }}{=}\{x, z: 0 \leqslant x \leqslant a, 0 \leqslant z \leqslant b\}$ :

$$
\begin{gathered}
\Delta \varphi=0, \quad(x, y) \in V_{1} \\
\left.\varphi\right|_{x=0}=\varphi_{0}(z),\left.\quad \varphi\right|_{x=a}=\varphi_{1}(z),\left.\quad\left(\frac{\partial \varphi}{\partial z}+B \frac{\partial \varphi}{\partial x}\right)\right|_{z=0, b}=0 \\
\varphi \in W_{2}^{1}\left(V_{1}\right), \quad \varphi_{\gamma} \in W_{2}^{1}[0, b], \quad \gamma=0,1 .
\end{gathered}
$$

Дивергенция вектора тока $J$ в параллелепипеде равна нулю [8], и нормальные токи на плоскостях параллельных оси $x$ равны нулю. Поэтому средний ток по сечению перпендикулярному оси $x$ не зависит от выбора сечения. Введем обозначения

$$
\left\langle J_{x}\right\rangle \stackrel{\text { def }}{=} \frac{1}{b} \int_{0}^{b} J_{x}(x, z) d z, \quad \bar{\varphi}_{\gamma} \stackrel{\text { def }}{=} \frac{1}{b} \int_{0}^{b} \varphi_{\gamma}(z) d z
$$

и определим магнетосопротивление $R(B)$ по формуле $R(B)=\sigma_{0}\left(\bar{\varphi}_{0}-\bar{\varphi}_{1}\right) / a\left\langle J_{x}\right\rangle$. Нетрудно показать, что сопротивление $R(B)$ выражается через потенциал $\varphi$ по формуле

$$
R(B)=\left(1+B^{2}\right) \frac{\left(\bar{\varphi}_{0}-\bar{\varphi}_{1}\right)}{a}\left\{-\frac{1}{b} \int_{0}^{b} \frac{\partial \varphi}{\partial x}(x, z) d z+\frac{B}{b}[\varphi(x, b)-\varphi(x, 0)]\right\}^{-1} .
$$

Магнетосопротивление $R(B)$ и средние значения $\bar{\varphi}_{0}, \bar{\varphi}_{1}$ измеряются в эксперименте. Мы ставим обратную задачу: восстановить потенциалы $\varphi_{0}, \varphi_{1}$ по магнетосопротивлению $R(B)$ и известным средним значениям $\bar{\varphi}_{0}, \bar{\varphi}_{1}$. Эта задача ранее не рассматривалась, поскольку функция $R(B)$ была неизвестна.

Недавно, в работе [9] для функции $R(B)$ была получена асимптотическая формула в предположении $b / a \ll 1$. Так, в случае $\varphi_{1} \equiv 0$ эта формула имеет вид

$$
R^{-1}(B)=1+\frac{\frac{b}{a} B^{2} a(B)-a^{0}(B) / \bar{\varphi}_{0}}{1-\frac{b}{a} B^{2} a(B)}+O\left(e^{-\pi a / b}\right) .
$$

Здесь функции $a(B), a^{0}(B)$ аналитичны в области $|B|<1$ и определяются формулами

$$
\begin{gathered}
a(B) \stackrel{\text { def }}{=} \sum_{k=0}^{\infty} B^{2 k}\left(\eta-\frac{1}{2}, D^{2 k+1} 1\right), \quad 0 \leqslant \eta \leqslant 1, \\
a^{0}(B) \stackrel{\text { def }}{=} \sum_{m=0}^{\infty}(-1)^{m} B^{m}\left(\varphi^{0}(\eta b)-\bar{\varphi}^{0}, D^{m+1} 1\right) .
\end{gathered}
$$

Асимптотической обратной задачей магнетосопротивления будем называть восстановление потенциала $\varphi_{0}(\eta b)$ по асимптотической формуле для $R^{-1}(B)$, полученной из формулы (36) отбрасьванием экспоненциально малых членов $O\left(e^{-\pi a / b}\right)$. 
Лемма 3. По функиии

$$
R_{0}^{-1}(B) \stackrel{\text { def }}{=} 1+\frac{\frac{b}{a} B^{2} a(B)-a^{0}(B) / \bar{\varphi}_{0}}{1-\frac{b}{a} B^{2} a(B)}
$$

в области $|B|<1$ и значению $\bar{\varphi}_{0}$ однозначно восстанавливается граничный потенииал $\varphi_{0}(z)$.

ДокАЗАТЕЛЬСТво. Функция $a(B)$ не зависит от потенциала $\varphi_{0}$ и определяется рядом (37). Функция $a^{0}(B)$ в силу формулы для $R_{0}^{-1}$ однозначно выражается через известные функции $a(B), R_{0}^{-1}(B)$. Коэффициенты разложения функции $a^{0}(B)$ в ряд Тейлора по $B$ определяются формулой (38). Из доказанной в $\S \S 1-2$ теоремы о полноте, ортогональности и ортонормируемости системы функций $D^{n} 1, n=0,1, \ldots$, следует, что

$$
\varphi_{0}(\eta b)-\bar{\varphi}_{0}=\sum_{n=1}^{\infty}\left(D^{n} 1\right)\left(\varphi_{0}(\eta b)-\bar{\varphi}_{0}, D^{n} 1\right) .
$$

Коэффициенты Фурье функции $\varphi_{0}(\eta b)-\bar{\varphi}_{0}$ по системе $D^{n} 1, n \geqslant 1$, в силу формулы $(38)$ равны коэффициентам степенного ряда Тейлора функции $a^{0}(B)$ по параметру $B$, умноженньг на $(-1)^{n-1}$. Значит, функция $\varphi_{0}(\eta b)-\bar{\varphi}_{0}$ однозначно восстанавливается по коэффициентам Тейлора функции $a^{0}(B)$ в области $|B|<1$.

\section{СПИСОК ЦИТИРОВАННОЙ ЛИТЕРАТУРЫ}

[1] Sz-Nagy B., Foias C. Harmonic Analysis of Operators on Hilbert Space. Amsterdam-London: North-Holland Publ. Company, 1970.

[2] Gohberg I., Krupnik N. One-Dimensional Linear Singular Integral Equations. V. 1: Birkhäuser Verlag, 1992.

[3] Зигмунд А. Тригонометрические ряды. V. 1, 2. М.: Мир, 1965.

[4] Кашин А. Б., Саакян А. А. Ортогональные ряды. М.: Наука, 1984.

[5] Лаврентьев М.А., Шабат Б. В. Методы теории функций комплексного переменного: Физматлит, 1987.

[6] Ramirez de Arellano E., Vasilevskii N. N. Algebras of singular integral operators generated by three orthogonal projections // Integr. Equat. Oper. Th. 1996. V. 25. P. 277-288.

[7] Девдариани Г.Г. Базисность одной системы синусов // Тр. ин-та прикл. матем. им. И.Н. Векуа. Т. 19. Тбилиси, 1987. С. 26-35.

[8] Ансельм А.И. Введение в теорию полупроводников. М.: Наука, 1978.

[9] Гуревич Ю. Г., Кучеренко В. В., Рамирес де Ареллано Э. О задаче с косой производной в теории гальваномагнитных эффектов // Матем. заметки. 1999. Т. 65. № 4. С. 520-532.

Departamento Matematicas, ESFM, IPN, Mexico

Поступило

Departamento Matematicas, CINVESTAV, IPN, Mexico

06.04 .1998

E-mail: valeri@esfm.ipn.mx, nvasilev@math.cinvestav.mx

Исправленный вариант

03.11 .1999 